\section{Feel great inside and out}

Patients are increasingly on the lookout for what does good and feels good, rejecting harsh chemicals and animal-based products in favour of plant power and herbal heroes. BE YOU gentle whitening toothpaste from Curaprox is the natural partner to their toothbrush - making them feel great inside and out.

Saying no to microplastics, triclosan, SLS and animal products, BE YOU celebrates herbal healing and promotes vegan vibes for those looking to bring pure joy to their day-to-day brushing. Chemical nasties give way to the power of glucose oxidase - an enzyme so natural that bees use it to keep germs away from their honey.

In this kind but mighty toothpaste, herbs and natural ingredients, such as extracts of echinacea, bitter orange, devil's claw and Indian pennywort, combine beautifully to nourish the mouth's tissue and help stop inflammation.

Available in six zesty, fresh flavours and in a collection of funky colours, let your patients try the power of plants in BE YOU toothpaste today.

For more information, call 01480 862084, email info@ curaprox.co.uk or visit www.curaprox.co.uk.

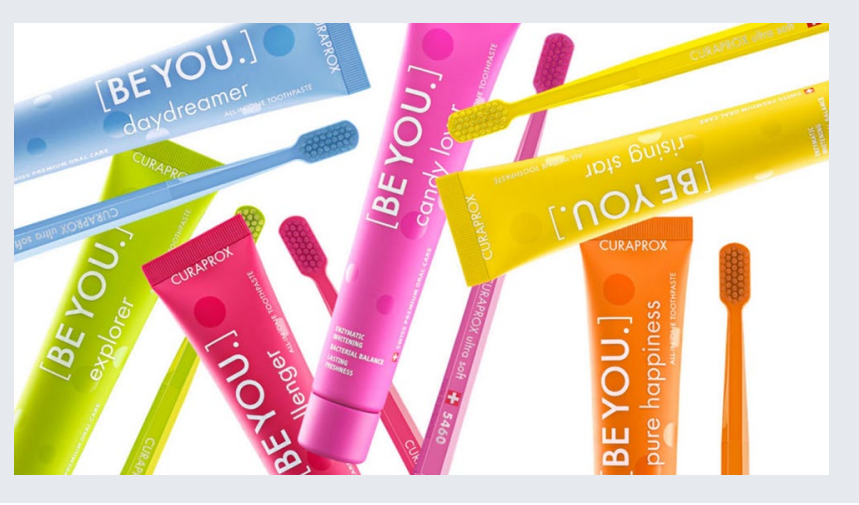

\section{Maximise the success of your GBT offering}

EMS Dental UK has just launched a new bespoke training package for practices invested in the guided biofilm therapy (GBT) protocol, to help maximise the success and potential of your GBT offering.

The training is tailor-made to your needs, held in your own practice and with your whole team, offering over six hours with one of the incredible SDA trainers, who will cover everything from the patient journey to marketing, as well as in-depth clinical benefits and technical support.

Once your training is complete, you are invited to become part of the exclusive GBT club, which offers strategies, hints and tips shared only with members.

Also available exclusively to club members is inclusion in the newly launched GBT practice finder website at https://www. switchtogbt.com, an exciting new tool for the public to search for their nearest GBT-certified provider, to meet increasing demand for GBT in the UK.

For more information, contact Education Manager, Scott Rogers, at srogers@ems-unitedkingdom.com or speak to your preferred GBT Expert.

\section{For dentists interested in full arch implants}

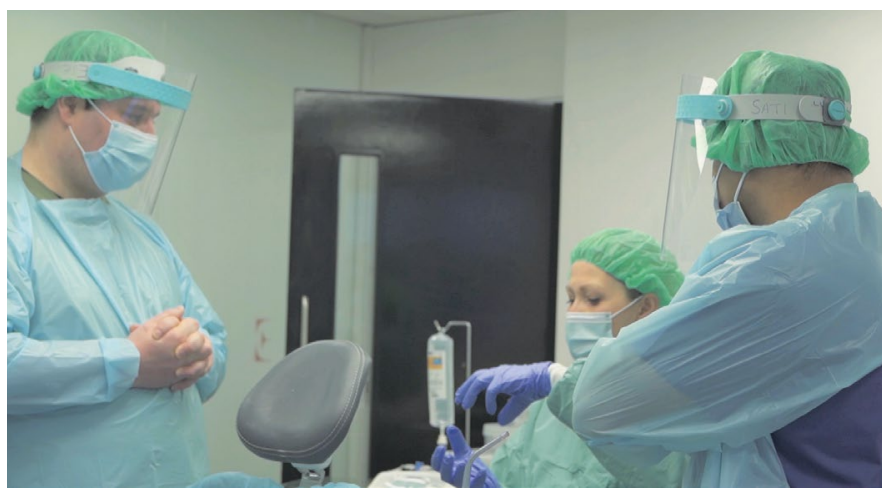

EvoDental, whose three clinics specialise exclusively in full arch implant rehabilitation, is continuing to welcome external dentists to watch as they treat a patient over the course of a day.

Since being set up at the start of 2021, EvoDental's free-of-charge 'EvoExperience' days have been attended by upwards of 75 dentists and the firm isn't stopping there. EvoDental are now encouraging more dentists to book in to see them as part of their 'access all areas' days, whereby visiting dentists follow the journey of a patient and watch their surgery, before seeing Evo's digital workflow in full effect and the patient's prostheses being milled and fitted.

Rudi Mukherjee, Clinical Director at EvoDental, said: 'We're absolutely delighted with how well received the EvoExperience has been. For years we have wanted to share our knowledge and expertise with the industry and our EvoExperience days have provided a fantastic outlet for this.

'We've seen dentists from across the UK come and spend a day with us - some with no implant experience at all and some who are placing full arch implants themselves - and we've been more than happy to answer any questions they have. We're really open here at EvoDental as we know that by working together, and collaborating, we're more likely as dental professionals to ensure the best possible outcome for patients.'

The EvoExperience, which comes with six hours' worth of CPD, can be booked by visiting: https://www.evodental.com/evoexperience/.

\section{Start your digital journey this September}

Join Planmeca on stand F40 at this year's Dentistry Show to find out just how easy it is to start your digital journey.

Across the two-day show, visitors to the Planmeca stand will have the opportunity to experience the wide range of digital solutions on offer including Planmeca ProMax 3D imaging unit, PlanMill $40 \mathrm{~S}$ milling unit and the Planmeca Emerald intraoral scanner. Planmeca's digital experts look forward to offering advice, product demonstrations and consultations on how their range of solutions can benefit practices, within modern-day dentistry.

Planmeca strongly believes in providing better care through innovation, and designs, builds and assembles its products using the latest technology and the best materials. If you're interested in digital dentistry then make Planmeca your no.1 destination at Dentistry Show London on 17-18 September. 